\title{
Evaluation methods for the autonomy of unmanned systems
}

\author{
WANG YueChao \& Liu JinGuo* \\ State Key Laboratory of Robotics, Shenyang Institute of Automation, Chinese Academy of Sciences, Shenyang 110016, China
}

Received December 8, 2011; accepted February 16, 2012

\begin{abstract}
Future military and civilian unmanned systems will be extensively used on land, under the sea, in air, and in space. Evaluation of their autonomy is critical to realize the autonomous operation ability of unmanned systems. This paper discussed the basic concepts of autonomy and the significance of autonomy evaluation, and then surveys and describes existing evaluating methods. Based on analyses of key technologies and these technologies' technical readiness levels, a cobweb evaluation model as a universal evaluation model has been used to evaluate unmanned systems. If the technology warps (aspects of autonomy) and wefts (levels of those aspects) of unmanned systems can be determined for the cobweb model, the model can handle the mutual coupling, high dimensions, and diversity of warp lines. This model potentially has wide application in evaluating various unmanned systems.
\end{abstract}

unmanned system, autonomy, evaluation, level, cobweb evaluation model

Citation: Wang Y C, Liu J G. Evaluation methods for the autonomy of unmanned systems. Chin Sci Bull, 2012, 57: 3409-3418, doi: 10.1007/s11434- 012-5183-2

In recent years, the successful applications of unmanned systems in the battlefield, space, the deep sea and other dangerous and distant environments have attracted much research interest. Unmanned systems include deep-space probes, spacecraft, unmanned air vehicles (UAV), unmanned ground vehicles (UGV), unmanned ships, unmanned submersibles, unmanned munitions, and unmanned sensors [1-7]. In the field of modern warfare and national security, unmanned systems not only perform reconnaissance, battlefield surveillance, and target tasks but also play an active role in electronic interference, air defense suppression, air combat, and ground attack. They have been the major fighting force to date and are expected to replace some manned systems ultimately, becoming the main force of combat and having more offensive and defensive attributes in the future. Almost all countries have paid great attention to the exploitation, development, testing, batch production and equipment of unmanned systems, assigned unmanned systems to an increasing number of missions, and regarded unmanned systems as crucial equipments for future tasks.

*Corresponding author (email: liujinguo@ sia.cn)
Since the working environment of an unmanned system is usually dangerous or remote, improving the autonomy of unmanned systems by advancing capabilities such as self-awareness, self-planning and self-control are important trends in the development of unmanned systems. Many organizations, including the decision makers of unmanned-system policy and the owners and users of unmanned systems (i.e. organizations involved in almost all aspects of a system's design and operation), are working on various evaluation methods and standards relating to unmanned systems [5-7].

In the research area of autonomy evaluation, Huang, a representational scholar from the United States, studied the autonomy-level framework of unmanned systems systematically [6-8]. In addition, related researches, such as that investigating the standard of unmanned platforms [5,9], the development roadmap of the unmanned air vehicle (UAV) autonomous control level [10-12], and mission planning at different autonomous levels [13], have resulted in many achievements. In recent years, scholars from Europe, Japan and other countries or regions have carried out extensive research into the autonomy control level of unmanned or robotic systems [14-20]. With the rapid growth of research 
on the autonomy control of unmanned systems in foreign countries, the autonomy control of unmanned systems has also attracted the attention of Chinese scholars. Research articles on the autonomy control level [21-28], autonomous control and autonomy-related technology [29-36], autonomy-related issues and other aspects [21,37-39] have been published. Experts in China's academic community investigated the autonomy control levels of the UAV and presented their findings at the Second Russian-Chinese Seminar on Unmanned Aerial Vehicles in 2005 [21], and many articles on autonomous control and autonomy were published by Chinese scholars at the First Chinese Navigation, Guidance and Control Conference in 2007 [22], the 2009 China Automation Association Conference [31], the Fourth Chinese Navigation, Guidance and Control Conference in 2010 [28], and the 2010 World Congress on Intelligent Control and Automation [38], where some papers were even presented as invited talks [28,31]. In addition, Chinese scholars organized a number of symposiums on autonomous control and autonomy. In August 2009, the Shenyang Institute of Automation, Chinese Academy of Sciences sponsored a high-level forum titled Autonomous Behavior of Mobile Robots and the Unmanned System. In August 2010, the Intelligent Automation Committee of the Chinese Automation Association sponsored a symposium titled Intelligent Control of Autonomous Systems. In October 2010, the Fourth Chinese Navigation, Guidance and Control Conference included a planetary session titled Advanced Control Technology of UAV. These symposiums and conferences presented the latest research results obtained in China.

Existing research has revealed that effective evaluation and relevant standards of the autonomy of unmanned systems play a leading role in the technological development of unmanned systems and guiding their development. Therefore, this paper discusses the basic concept of autonomy and the significance of autonomy evaluation. We then investigate and analyze existing evaluation methods. Finally, according to analyses of key technologies and technical readiness levels, we use a universal model, the cobweb evaluation model, to evaluate the autonomy of unmanned systems. We hope that the cobweb model will be used and discussed by colleagues.

\section{Definition of autonomy}

The terms autonomy and autonomous have wide application, and are used in many fields with various meaning. Their descriptions differ for different objectives. This paper focuses on autonomy intently defined for unmanned systems.

Research on unmanned systems has found that the main reason why unmanned systems can work without an operator is that they are capable of self-management; i.e. they have certain autonomous ability or autonomy. Therefore, we first need to define the meaning of autonomy when we research the autonomy of an unmanned system. Autonomy has been defined in many related studies, in which the definition proposed by the Autonomy Levels for Unmanned Systems (ALFUS) working group of the National Institute of Standards and Technology (NIST) being the most comprehensive and standardized. The working group was established in July 2003 and includes the U.S. Department of Defense, Department of Commerce, Department of Transport, and Department of Energy and their customers. The group's objectives are to establish methods for establishing metrics of autonomy and to draft a framework for autonomy levels of unmanned systems. They defined the autonomy of an unmanned system (UMS) as follows [6].

“A UMS's own ability of sensing, perceiving, analyzing, communicating, planning, decision-making, and acting, to achieve its goals as assigned by its human operator(s) through designed human-robot interaction (HRI). Autonomy is characterized into levels by factors including mission complexity, environmental difficulty, and level of HRI to accomplish the mission."

From this definition, we see that autonomy is the unmanned system's capability of being independent of an operator and being self-managed. Its resemblance with definitions proposed by many other autonomy researchers is that autonomy can be achieved by four basic functions: perception (supervision), orientation (diagnosis), solution (planning) and action (implementation) [40]. The definition of autonomy differs for the different application areas of unmanned systems. Ref. [41] defined autonomy in two dimensions: self-direction and self-management. In [42], autonomy was defined as a measurable value that can be used to distinguish various autonomy levels. We consider the ALFUS definition in [6] to be the most suitable and we thus state the following.

(1) The autonomy of unmanned systems is the performance achieved by unmanned systems when they use their hardware and software platforms to complete a given mission in a dynamic and unstructured environment, in terms of mission success and efficiency, through self-awareness and analysis of their situation, self-learning and self-decisionmaking, while minimizing human involvement.

(2) Autonomy is comprehensively determined by the key technologies that determine the ability of the system to realize the above-mentioned behaviors.

\section{Significance of autonomy evaluation}

Increasing the autonomy of unmanned systems is an important trend in the development of unmanned systems. Methods of evaluating autonomy are important to government policy makers and the owners, designers and users of unmanned systems. A quantitative assessment of the autonomy of unmanned systems can avoid ambiguity in wording and performance description. It can also improve 
the science and operability of unmanned systems. Additionally, quantitative assessment is conducive to project implementation and gradual performance improvements.

First, evaluations can help the planning researcher and decision maker quantify the autonomy level of unmanned systems, and index the developments and achievements of unmanned systems from low to high. For example, the autonomous control levels attained in three roadmaps of unmanned air vehicle development have been useful in UAV research planning in the United States [10-12].

Second, before or during product development, the designer of unmanned systems should consider the product evaluation problem and choose or develop a multi-disciplinary and/or multi-target evaluation method. Sometimes, a novel method is needed to measure the performance of a product and to determine whether product targets will be met. For example, the US Air Force and Boeing X-45 project team DARPA proposed methods to measure the autonomy of their products [43].

Finally, the user of unmanned systems requires a set of standards or methods that can evaluate the important properties of many similar products. Currently, almost all unmanned air vehicles within the U.S. military have their own Autonomous Control Level (ACL) values to indicate their autonomy.

\section{Survey of current evaluation methods}

Researchers around the world have carried much research into the evaluation of unmanned systems, each having their own clear application objectives [6-12,40-55]. The level of research interest in China on this topic has slowly risen, with existing research mainly having focused on introducing, analyzing, and tracking foreign research; Chinese research is thus still in its infancy [21-25,27,28,30,32,33, $37,38]$. Our review of the existing evaluation methods of the autonomy of unmanned systems has found that the evaluation methods mainly include but are not limited to the level evaluation method, double-axis method, three-axis method, look-up table method and formulation method. The following presents characteristics of these methods and some representative examples.

\subsection{Level evaluation method}

The level evaluation method usually classifies the autonomy of a system into one of many levels according to the system's performance. These levels are numbered, such as $0-10$ or $10-0$. Among level evaluation methods, Sheridan's Levels of Automation is typical. The levels were first produced in 1991 [44] and then modified in 2000 [45], and they are listed in Table 1. Although this method cannot be applied to unmanned systems directly, most unmanned systems are automatic devices, and computers are the hardware basis of unmanned-system autonomy. This method therefore has some applicability to the autonomy quantification of unmanned systems. Since it only considers simple factors, it often only reflects one aspect of the unmanned system's performance.

Another representative example of the level evaluation method is the autonomy level evaluation formulated by the National Aeronautics and Space Administration (NASA) for their aircraft systems. NASA's Vehicle Systems Program High-altitude Long-range Sector (HALE Sector) [46] analyzed the ACLs of the U.S. Department of Defense/Air Force Research Laboratory, and they deemed that the ACLs were too many and too specific for the long-range science missions of desired altitude and thus used a streamlined autonomy evaluation, as presented in Table 2.

Table 1 Sheridan's levels of automation [44]

\begin{tabular}{|c|c|}
\hline Level & Definition \\
\hline 1 & The computer offers no assistance, human must do it all \\
\hline 2 & The computer offers a complete set of action alternatives, and \\
\hline 3 & Narrows the selection down to a few, or \\
\hline 4 & Suggests one, and \\
\hline 5 & Executes that suggestion if the human approves, or \\
\hline 6 & $\begin{array}{l}\text { Allows the human a restricted time to veto before automatic } \\
\text { execution, or }\end{array}$ \\
\hline 7 & Executes automatically, then necessarily informs the human, or \\
\hline 8 & Informs him after execution only if he asks, or \\
\hline 9 & Informs him after execution if it, the computer, decides to \\
\hline 10 & $\begin{array}{l}\text { The computer decides everything and acts autonomously, ignor- } \\
\text { ing the human }\end{array}$ \\
\hline
\end{tabular}

Table 2 NASA's autonomy levels for aircraft systems in the HALE sector [46]

\begin{tabular}{|c|c|c|c|}
\hline Level & Appellation & Description & Character \\
\hline 0 & Remote control & People in the loop remote control flight (100\% human involvement) & Remote control aircraft \\
\hline 1 & Sample automatic operation & $\begin{array}{l}\text { Under the surveillance of the operator, perform the task relying on } \\
\text { self-control auxiliary equipment }(80 \% \text { human involvement })\end{array}$ & Autopilot \\
\hline 2 & Remote operation & $\begin{array}{l}\text { Execution the program task which the operator written ( } 50 \% \text { human in- } \\
\text { volvement) }\end{array}$ & $\begin{array}{l}\text { UAV integrated management the } \\
\text { pre route takeoff point }\end{array}$ \\
\hline 3 & $\begin{array}{l}\text { Highly automated } \\
\text { (semi-autonomous) }\end{array}$ & $\begin{array}{l}\text { Have some situational awareness ability, can perform complex task auto- } \\
\text { matically, and make their routine decision-making ( } 20 \% \text { human involve- } \\
\text { ment) }\end{array}$ & $\begin{array}{l}\text { Continue the task after automatic } \\
\text { takeoff / landing link interrupts }\end{array}$ \\
\hline 4 & Complete autonomy & $\begin{array}{l}\text { Have the ability of perceiving body and environmental widely, the ability } \\
\text { and authority to make a comprehensive decision }(<5 \% \text { human involvement) }\end{array}$ & Automatic re-planning task \\
\hline 5 & Co-operation & Teamwork between the number of UAVs & Cooperation and coordinated flight \\
\hline
\end{tabular}


The autonomy of NASA aircraft is divided into six levels, from the lowest level 0 to the highest level 5. To determine the autonomy level, NASA used intuitive data, namely the length of time of human involvement in the operation of an unmanned vehicle, with less control time indicating higher autonomy of the UAV and greater self-management ability; this is the most obvious feature of the autonomy levels of the HALE Sector. However, because the scale is simple and intuitive, some factors have been ignored.

\subsection{Double-axis method}

The two axes of the double-axis method are the autonomy axis and time axis. The autonomy axis is marked with an autonomy level and connotation, while the time axis is marked with the development era of autonomy. Employing the double-axis method, we clearly see the autonomy level of the specific unmanned systems at a specific time. This approach helps researchers and government departments to formulate research planning and decisions. Among double-axis methods, the ACL method proposed by the U.S. military is the most representative, and it is shown in Figure 1 [10]. The ACL method categorizes the autonomy of the system into one of 10 levels, namely remote guidance, real-time health diagnosis, adaptation to failures and flight conditions, route re-planning of the aircraft, group coordination, group tactical re-planning, group tactical goals, distributed control, group strategic goal, and fully autonomous swarms.

The definition of autonomy implies that autonomy is the self-management ability of the system, and this kind of ability can be classified from strong to weak. Autonomy levels obviously quantify the strength of autonomy. The interaction information required by the unmanned system is inversely proportional to this quantification. The more interaction information the unmanned system requires from the outside, the more the unmanned system is dependent on the operators, and its autonomy level is lower. If an unmanned system is controlled completely from the outside, then it is simply a remotely controlled system.

The autonomy level of the unmanned system ultimately reflects the ability of an unmanned system(s) to complete a mission without operator assistance, such as an attack mission carried out by multiple unmanned systems. Here we provide a simple analysis and introduction of the ACL [10-12]. Taking a UAV as an example, below ACL 3, systems pursue remote-control operation, while above ACL 3, systems pursue preliminary fight operation, such as firing weapons. For example, the roadmap produced in 2000 set the goal of ACL 3 for the X-45 developed in 2005. At ACL 4 , the fire control ability of the single unmanned system reaches its highest level. This illustrates why project realization can go hand in hand with theoretical research at the same level. On the other hand, performance at ACLs 1-3 has improved with time. ACL 4 expresses the highest performance of an individual system. ACLs 5-10 express

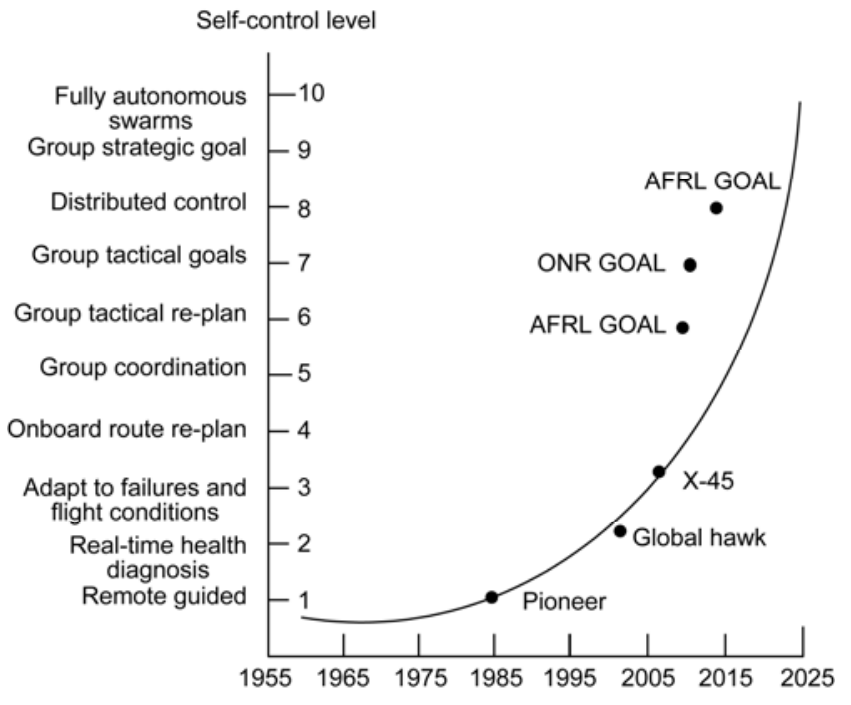

Figure 1 Department of defense's levels of autonomy [10].

the characteristics of groups; however, the lack of specific meaning for cooperation has led inconsistent classification at ACLs 5-10; for example, cooperation can refer to an air-to-ground attack or reconnaissance. However, there is no doubt that ACL 10 involves UAV group warfare, and the highest fight mode of the unmanned systems is an attack by multiple unmanned systems rather than a single unmanned system attack, which is the same as the combat situation for a manned vehicle.

\subsection{Three-axis method}

The three-axis method usually considers three aspects of system autonomy, and determines the different levels that the system can reach. The overall level is obtained by weighting these grades. This approach considers more factors of system autonomy than the above methods, but the coupling and independence between these factors are difficult to determine. A representative three-axis method, as shown in Figure 2, is ALFUS researched by Huang et al. [6] at the Intelligent Systems Division of NIST.

The ALFUS working group proposed a model for evaluating the autonomy level considering three main factors, and used it to measure the autonomy of unmanned systems. These three factors are the complexity of the environment in which tasks are performed, the level of dependence on the outside and the complexity of all missions conducted by the system. The working group plotted these factors of the autonomy level on three axes, with separate axes having a set of metrics that measure the complexity of the environment, the level of dependence on the outside or the mission complexity. As shown in Figure 2, we can comprehensively evaluate the autonomy levels of unmanned system using the three-dimensional coordinate method. The main idea is that when assessing the autonomy levels of unmanned systems, we decompose the tasks to perform, execute the sub-tasks at 


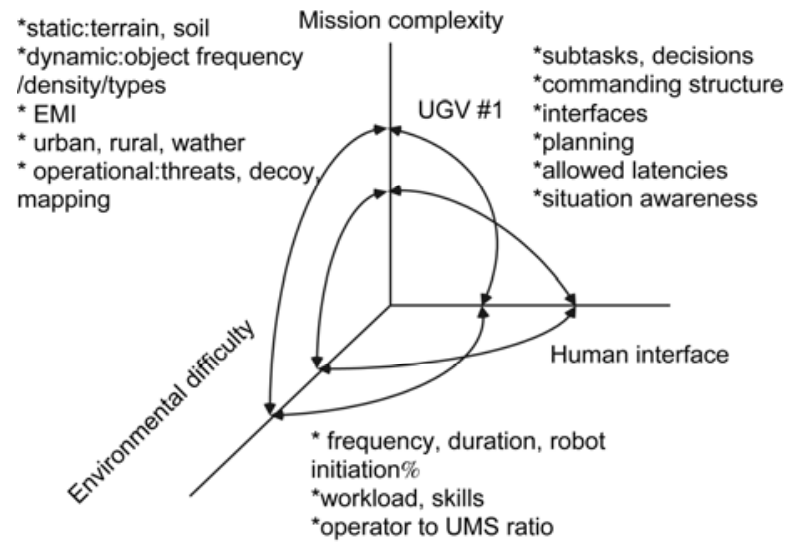

Figure 2 Detailed model of ALFUS [6].

the bottom level, assign weights according to the measurement of the three factors in the model, and average the sum of the weights for the tasks at the same level. As the mission whose level in the top, we should calculate in turn until we obtain the autonomy level of the top task. In practice, however, there are many problems with this model that cannot be ignored. For example, how should we measure the factor for each axis, and how should weights be allocated? Additionally, it is an open question how to assess the autonomy level of the system. There are many scheme options, including calculating the maximum/minimum value after summing the weights, and taking the average of the weights. Different schemes may produce different evaluation results.

\subsection{Table lookup method}

The table lookup method is a multi-dimensional evaluation method that usually sets out the level of autonomy, evaluation parameters and evaluation indicators of the system performance in a table. The method can describe the autonomy level of the system in more detail in all respects, but the same system will have different values for different evaluation parameters. The three-dimensional (3D) intelligent space evaluation method of Draper Laboratory is one such method, and it is presented in Table 3 [52,53]. Draper Laboratory has developed robotic systems for a number of years, and they attempted to measure the performance of their robotic systems in various tasks. The Naval Research Office has provided some required indexes. Using these indexes, the researchers at Draper Laboratory established four levels to evaluate the autonomy of the system in terms of mobility control, task planning and situation awareness. The 3D intelligent space diagram constructed by researchers at Draper Laboratory is described briefly here. Their standards included several different options for classification. Table 3 is an overview of the 3D intelligent space diagram, which has two unique features. (i) There are three indexes, and we can use three-axis charts to represent the results for the benefit of management. (ii) It has an option related to operational issues.

Using a 3D intelligence space table is the simplest way to make a one-sided assessment of the success or failure of the assessment system. First, it establishes the development space of a task, and many factors can be confirmed through in-depth analysis of the assignment. We then use collaboration, situation awareness and uncertainty situation planning as the three aspects to express the extent of the mission, and add many factors for each aspect to determine the grade of the system. The situations of the three aspects are then combined, and an ideal system determination method is established.

\subsection{Formulation method}

A formulation method usually calculates the autonomy level of the system directly using a multi-variable function. There are usually two kinds of multi-variable functions: the product type and the weighted type. There are usually many demands of the design of the function, the choice of the variables and the confirmation of the weights/indices. Establishing multi-variable functions usually requires much experience and data, and it is specific system. One representative work was carried out by Curtin et al. [48], who defined the autonomy and intelligence of an underwater robot as

$$
\begin{aligned}
\text { Autonomy }= & C_{n}\left[\frac{\text { Control bits }}{\text { Total message size }}\right]^{-i} \\
& \times\left[\frac{\text { Control time }}{\text { Total mission time }}\right]^{-j}, \\
\text { Intelligence }= & C_{x}\left[\frac{\text { Mission completion time }}{\text { Maximun endurance }}\right]^{-m} \\
& \times\left[\frac{\text { Achieved result }}{\text { Objective }}\right]^{-n},
\end{aligned}
$$

\begin{tabular}{|c|c|c|c|}
\hline \multicolumn{4}{|c|}{ Metric } \\
\hline Level & Mobility control & Task planning & Situational awareness \\
\hline 1 & None, RPA Only & None, RPA Only & None, RPA Only, or sensor as conduit \\
\hline 2 & Operator Assisted & Waypoint or feature oriented & $\begin{array}{l}\text { Low-level sensor processing, e.g. visual } \\
\text { serving (template tracking) }\end{array}$ \\
\hline 3 & Get to waypoint, do one feature-based command & Interpret goals into action & Single-sensor model matching \\
\hline 4 & Integrate multiple actions & Multi-Agent Collaboration and C2 & Integrated, multi-sensor fusion \\
\hline
\end{tabular}

where $C_{n}$ is an index of the task communication and $C_{x}$ is an index of task complexity.

Table 3 3D intelligence space [52] 
The advantages of this definition are that it has the ability to quantify and standardize the performances of different systems. This method is used to evaluate the collision avoidance performance of many unmanned systems. Baseline data are initially obtained for this purpose, and more and more operation tasks are subsequently completed and their data used in the evaluation. In addition to the relative assessment, the intelligence and autonomy evaluation of the AUV is also important in determining the system's legal responsibility.

Doboli et al. [55] at the State University of New York proposed a new calculation method to judge autonomy. They believe that the quantity and quality of autonomy depends on human inputs, which must ensure the correct operation of the system in a widely changing space; however, the rule needs a specific geographic area and some time to adapt.

\subsection{Extension of classic methods}

Among the abovementioned evaluation methods, several classical methods have been widely applied and cited, including Sheridan's LOA, the US military's ACL, and Huang's ALFUS.

Researchers have extended the above evaluation methods to various degrees. For example, using ALFUS, Yang and Zhang [25] put forward the assessment of the autonomy level of unmanned systems by considering four aspectsthe degree of change in the environment, the degree of task completion, the degree of state stability of the system, and the degree of human-computer interaction-and then introduced fuzzy theory to this method by quantifying the autonomy level of unmanned systems with a fuzzy decision. From analysis of the mechanism of human intelligence and with in- depth study of the autonomy control-level classification of the UAV, Chen et al. [28] proposed a nine-level evaluation of the autonomy control of an unmanned system, and then enriched the content of the autonomy control evaluation level with an autonomy function, autonomy types, intelligence attributes, information sharing capabilities and other aspects. Suresh and Ghose [20] carried out in-depth research on 11 levels of autonomy control from single-system autonomy to group autonomy, and they provided detailed explanations of each level through published extensive literature on autonomy. Moreover, they divided each level into several sub-layers, and they believed that communication and information would play a key role in realizing the autonomy of an UAV. All these extended research works have greatly expanded the content and scope of the autonomy evaluation method.

\section{Preliminary investigation of a universal evaluation method}

A quantitative evaluation method of unmanned system au- tonomy is important, but technological gaps, cultural differences, technology confidentiality and other reasons may be prohibitive to its development. In fact, this problem has existed for a long time, especially in terms of the introduction of advanced foreign technology. In addition, various research organizations or institutions use the same word with different meanings when considering their evaluation methods in different ways. Therefore, proposing an effective universal approach is an important challenge. The solution to this problem requires the joint efforts of internal and external colleagues.

\subsection{Brief introduction to the cobweb evaluation model}

Assessing the autonomy level of unmanned systems relates to evaluating the autonomy performance of a system in completing a designated mission. Therefore, for different tasks, the autonomy level of an unmanned system differs; i.e. the level is relative, not absolute one. To describe this dynamical change, we use a cobweb evaluation model here. It is should be noted that the cobweb model presented here differs from that used in economics. The proposed model has an original point with several radiating axes. Each axis represents an evaluation aspect, which is the key technology that determines an unmanned system's autonomy. Each aspect has nine levels (1-9), and these levels indicate technology maturity. For each unmanned system, there is a corresponding mapping to a level of each aspect of evaluation, and the corresponding level for each axis constitutes the cobweb's latitude. Thus, we can evaluate the unmanned system's autonomy, as shown in Figure 3(a). Let us take a UAV system as an example. The aspects of the evaluation (key technologies) in this example are only navigation, reconnaissance, attack, collaboration and interaction. We assume that the autonomy level of navigation is 3 , the level of reconnaissance is 2 , the attack level is 2 , the collaboration level is 3 , and the interaction level is 1 . The autonomy evaluation cobweb model is then constructed as shown in Figure 3(b).

Using the descriptive method shown in Figure 3, we can build autonomy cobweb evaluation models of various unmanned systems such as UAVs, unmanned undersea vehicles, unmanned surface vehicles, unmanned ground vehicles, military robots, military satellites, planetary exploration robots, unmanned systems for handling munitions and unmanned sensors, determining the number/value of the latitude through experience or from experimental data. Since unmanned systems used in different working environments such as on land, under the sea, in air, and in space have different characteristics, we must correctly handle the mutual coupling, high dimensions, diversity and other complex factors in designing evaluation models, and research dynamic optimization theories and methods in model design. Moreover, the model can be gradually improved by comparing numerical simulation results and real model tests. 

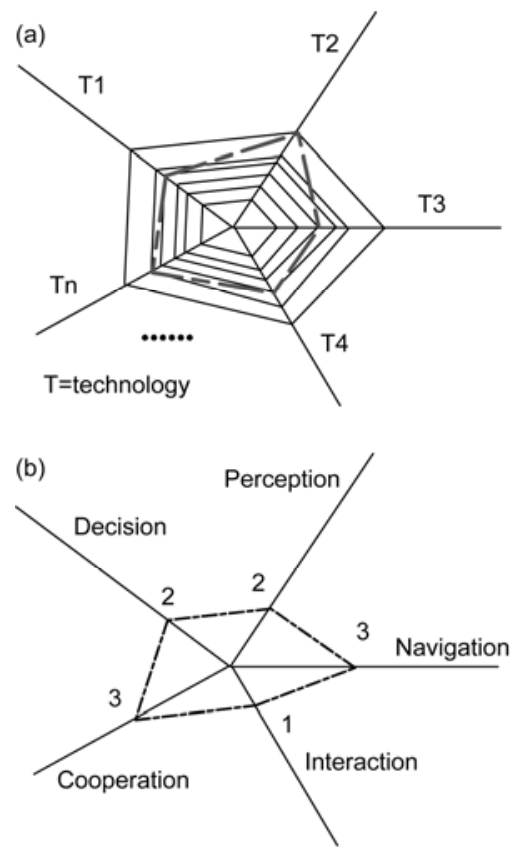

Figure 3 Cobweb evaluation model of autonomy.

\subsection{Key technologies of the cobweb evaluation model}

The key technologies deciding the autonomy of unmanned systems are the bases of the development of such systems. Research and development on unmanned systems relate with many professional disciplines but can roughly be divided into two types of technologies: common technology supporting the development of unmanned systems and expertise technology for special applications of unmanned systems. The two jointly promote the development and application of unmanned systems.

Some common technologies and expertise technologies of unmanned autonomous systems have attracted researchers' wide attention, such as sensing technology $[54,56]$, human-robot interaction technology [45], information communication technology [20], path planning technology $[32,57,58]$, multi-platform collaboration technology $[15,35$, $36,50,59,60]$, movement and motion-control technology[17, 61,62], autonomy energy technology [39], mission planning technology $[13,16,63]$, and autonomous flight-control technology $[31,34,64,65]$. Ref. [1] comprehensively overviewed key technologies required for a variety of unmanned combat systems. Ref. [43] suggested that technologies relating to planning and decision-making, sensing and understanding, detection and diagnosis, networking and collaboration and human-robot interaction are common key technologies of unmanned autonomous systems. Ref. [66] proposed that intelligent software, sensing, navigation, communications, energy, and the ability to survive provide greater challenges to a system's autonomy. Ref. [4] generalized that the key technologies of the unmanned ground vehicle include those relating to sensing, navigation, planning, adaptation, power and energy, human-robot interaction, behavior and skills, maintenance, and communications. Ref. [2,67] discussed the key technologies of surface and underwater unmanned systems, which included those relating to intelligent planning and decision-making, intelligent control, underwater target detection and recognition (pattern recognition), sensors and information processing, autonomous navigation, energy and propulsion, system simulation, communication and networks for the unmanned autonomous underwater platform, while wireless data communications technology, integrated drive power, autonomous control technology, surface target detection and self-recognition, and autonomous navigation technology for unmanned autonomous surface platforms. Ref. [33] proposed that the key technologies of a UAV autonomous control system should include those relating to architecture, perception and cognition, planning and control, coordination and interaction. Research has shown that several main types of unmanned systems, such as unmanned ground systems, unmanned undersea/surface systems, unmanned aerial systems and unmanned satellite systems have common technologies in terms of autonomy evaluation.

Many techniques are needed to select key technologies in the cobweb model of autonomy evaluation. On one hand, we can learn lessons from existing research on key technologies; on the other hand, the approach should suit the specific case. We can select the key technologies of the autonomy evaluation model by comparing technologies for the same type of mission, the same type of platform or the same type of technology, as in the following.

(1) For the same mission type, we can choose platforms that have different degrees of technological maturity, and the autonomy of these platforms will differ. Alternatively, for the same mission type, with time, the same type of platform will improve with developments in technology and autonomy.

(2) For the same type of platform, according to the different technologies available at different times, the degree of technological maturity and autonomy can change.

(3) For the same time, platforms of the same type will have individual differences in terms of technical maturity and autonomy for the same mission.

\subsection{Level of technological readiness}

Applying the existing Technology Readiness Levels (TRLs) is a practical approach for evaluating key technologies. As shown in Figure 4, the TRLs vividly describe the degrees of maturity of specific technologies, the development status and technical risk of the specific technologies [68]. The TRL is sometimes called the Technology Maturity Level in [70].

The TRL evaluation method was first introduced by NASA for space project management, and then widely used by the United States, Britain, Europe and other countries 


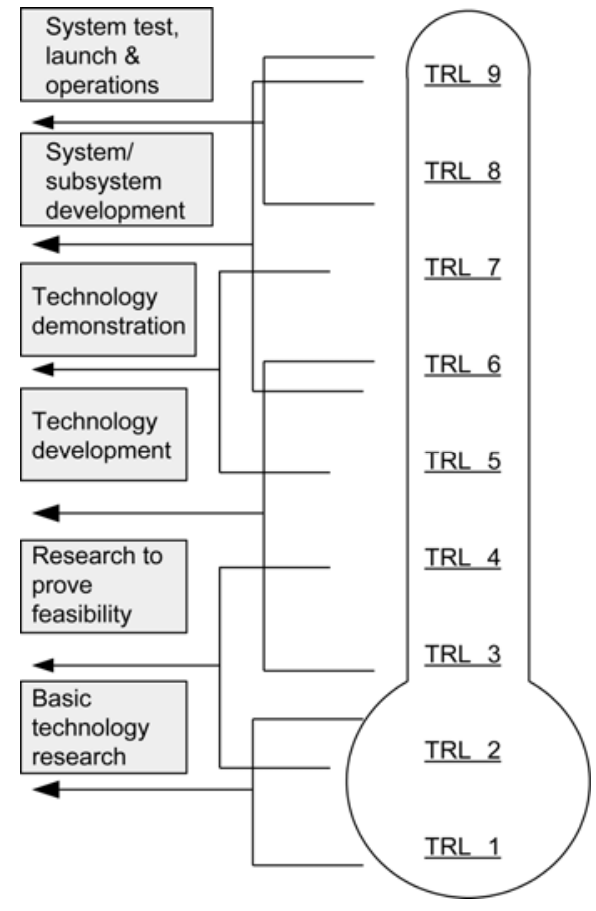

Figure 4 Chart of TRLs ${ }^{1)}$.

and regions. Refs. [70,71] gave a comprehensive discussion of the TRL. Chinese scholars have also carried out much research on the TRL; e.g. Qian et al. [72] used the TRL to evaluate underwater warfare equipment, Chen et al. [73] used it in the development of missiles, and Zhu et al. [74] suggested that the TRL be applied to the development of spacecraft.

In the cobweb evaluation model of autonomy, the evaluation of the technical maturity of key technologies can learn from the TRL evaluation method. There has been in-depth research on how to apply the TRL evaluation method; e.g. refs. $[68,69]$ gave authoritative requirements and an overview, and ref. [72] gave a typical example of primary application. In addition, version 2.2 of the TRL's calculator software has been developed and is freely available from a U.S. website ${ }^{2)}$. There are detailed descriptions of the TRL calculator in [75,76], and it is therefore not described here.

\section{Discussion and purpose}

The autonomy of unmanned systems in unstructured, and dynamic environments is becoming an important research topic and is critical for applications in fields such as scientific exploration, national security, public safety, and social services. Many authorities have employed quantitative evaluation methods of the autonomy of unmanned systems, and such studies carried out by research project planners, project decision makers, developers and users are important to both fundamental theoretical research and engineering application research. Although it is well known that quantitative evaluation of the unmanned system is important, there are still many problems with the adaptability of existing evaluation methods, which need to be improved for general utilization. The autonomy evaluation method of the unmanned system must consider the diversity, multidimensionality, hierarchy, and primary and secondary natures of the application target and the system itself, so that it can avoid ambiguity in the formulation and description of the autonomy of autonomous unmanned systems. Owing to technological gaps, cultural differences, security classification and other reasons, it is difficult for researchers to exchange ideas.

On the basis of our understanding of autonomy evaluation methods, this paper established a universal model for autonomy evaluation. The multi-dimensional cobweb model of autonomy evaluation used in this paper improves on the science and operation of existing evaluation methods. The model can be used as an important reference in the engineering implementation of unmanned systems and can gradually help increase autonomy performance. In China, research on the evaluation of unmanned systems' autonomy has only recently started, and there is an urgent need for long-term support from relevant government departments. The authors believe that with the development of autonomy theory and autonomous technology, the unmanned system will have wide application and be more standardized and intelligent in the near future.

This work was supported by the National Security Program on Key Basic Research Projects (61381) and the CAS President's Award Winner Foundation. The authors express their thanks to Professor Feng XiSheng, Academician of the Chinese Academy of Engineering, Dr. Xu HongLi, Associate Professor at the Shenyang Institute of Automation, and the anonymous reviewers for their valuable comments.

1 Lin C Y, Zhang Y Q. Intelligent Unmanned Combat Systems (in Chinese). Changsha: National University of Defense Technology Press, 2008

2 Jiang X S. Underwater Robots (in Chinese). Shenyang: Liaoning Science and Technology Press, 2000

3 Antsaklis P J, Passino K M, Wang S J. An introduction to autonomous control systems. IEEE Contr Syst Mag, 1991, 11: 5-13

4 Research Council of the National Academies. Technology Development for Army Unmanned Ground Vehicles. Washington DC: The National Academic Press, 2002

5 ASTM F2541-06, Standard Guide for Unmanned Undersea Vehicles (UUV) Autonomy and Control, 2006

6 Huang H M, Albus J, Messinan E, et al. Specifying autonomy levels for unmanned systems: Interim report. In: Gerhart G R, Shoemaker C M, Gage D W, eds. Proceedings of the 2004 SPIE Defense and Security Symposium Conference. 2004 Apr 12-14. Orlando, Florida: Unmanned Ground Technology CI Press, 2004. 386-397

\footnotetext{
1) Definition of Technology Readiness Levels. http://esto.nasa.gov/files/TRL_definitions.pdf, 2010

2) TRL Calculator Upgrade to v 2.2. https://acc.dau.mil/communitybrowser.aspx?id=25811
} 
7 Huang H M, Pavek K, Novak B, et al. A framework for autonomy levels for unmanned systems (ALFUS). In: Association for Unmanned Vehicle Systems International, eds. Proceedings of the AUVSI's Unmanned System North America Conference, 2005 Jun 28-30. Baltimore: Arlington Va Press, 2005. 849-863

8 Huang H M. Autonomy Levels for Unmanned Systems (ALFUS) framework: Safety and application issues. In: Messina E, Madhavan $\mathrm{R}$, eds. Proceedings of the Performance Metrics for Intelligent Systems Workshop, 2007 Aug 28-30, Washington DC USA, ACM New York NY USA Press, 2007. 48-53

9 ASTM E2521-07, Standard Terminology for Urban Search and Rescue Robotic Operations, 2007

10 Office of the Secretary of Defense. Unmanned Aerial Vehicles Roadmap 2000-2025. Technical Report, Department of Defense, Washington DC, 2001

11 Office of the Secretary of Defense. Unmanned Aerial Vehicles Roadmap 2002-2027. Technical Report, Department of Defense, Washington DC, 2002

12 Office of the Secretary of Defense. Unmanned Aircraft System Roadmap 2005-2030. Technical Report, Department of Defense, Washington DC, 2005

13 Brent A, Rosemary D P, Robert J S. Mission planning system for vehicles with varying levels of autonomy. US Patent, US7765038B2, 2010-7-27

14 Satoshi K, Koichi N, James K, et al. Low-level autonomy of the humanoid robots H6 \& H7. In: Jarvis R A, Zelinsky A, eds. Robotics Research STAR 6. Heidelberg: Springer, 2003. 83-97

15 Jeremi G, Simon L. Embedding heterogeneous levels of decisional autonomy in multi-robot systems. In: Alami R, Chatila R, Asama H, eds. Distributed Autonomous Robotic Systems 6. Heidelberg: Springer, 2007. 263-272

16 Hervé A, Frederic D, Magali B, et al. Goal driven planning and adaptivity for AUVs. In: Proceedings of the First Workshop on Control Architectures of Robots, Montpellier, 2006 Apr 6-7, 191-197

17 Broten G S, Collier J A, Giesbrecht J L, et al. Architecture for autonomy: Implementation and usage on the raptor UGV. Technical report, Defence R\&D Canada-Suffield, 2006

18 Protti M, Barzan R. UAV autonomy - which level is desirable? which level is acceptable? alenia aeronautica viewpoint. In: Proceedings of the AVT-SCI Joint Symposium, France, 2007 Nov 19-21. 1-12

19 Fil M. The test and evaluation of unmanned and autonomous systems. Int Test Eval Assoc J, 2008, 29: 388-395

20 Suresh M, Ghose D. Role of information and communication in redefining unmanned aerial vehicle autonomous control levels. P I Mech Eng G-J Aer, 2010, 224: 171-197

21 Gao J S, Chen X D, Yang H. UAV autonomy research (in Chinese). In: Chinese Society of Aeronautics and Astronautics, eds. Proceedings of the second China-Russia Symposium on UAV, 20054 12-15, Beijing. Beijing University of Aeronautics \& Astronautics Press, 2005. 97-102

22 Gao J S, Ding Q X, Zou Q Y. Preliminary analysis of the foreign quantitative assessment methods of UAV's autonomy(in Chinese). In: Proceedings of the First China Navigation, Guidance and Control Conference, 200711 4-5, Beijing. 722-726

23 Chen H, Wang X M, Zhao K R. Hierarchical control architecture of autonomous control for unmanned combat aerial vehicles (in Chinese). Acta Aeronaut et Astronaut Sin, 2008, 28(Suppl): 224-228

24 Gao J S, Yu F, Ji X G. Current situation of studies on autonomous control level of UAVs (in Chinese). Electron Optics Contr, 2009, 16: 51-54

25 Yang Z, Zhang R B. Fuzzy evaluated method for the autonomy levels of unmanned systems (in Chinese). J Chin Comput Syst, 2009, 30: 2043-2047

26 Chen H, Wang X M, Li Y. A survey of autonomous control for UAV. In: Deng H P, Wang L Z, Wang F L, et al., eds. Proceedings of the International Conference on Artificial Intelligence and Computational Intelligence, 2009 Nov 7-8, Shanghai, China. Springer Press, 2009. 267-271

27 Gao J S, Wang Z Y, Chen X D. Study on UAV's autonomous control level (in Chinese). Aeronaut Sci Tech, 2010, 2: 40-43

28 Chen Z J, Wei J Z, Wang Y X, et al. UAV autonomous control levels and system structure (in Chinese). Acta Aeronaut et Astronaut Sin, 2011, 32: 1075-1083

29 Zhou R, Li R F, Chen Z J. Autonomous control of unmanned tactical aircraft (in Chinese). Contr Decis, 2001, 16: 344-346

30 Wang Y X, Cai Z H. Autonomous flight control of unmanned aerial vehicle (in Chinese). Aeronaut Manuf Tech, 2009, 8: 26-31

31 Wang Y C, Qi J T, Han J D. Research on advanced control algorithms for flying robot with rotary wing (in Chinese). In: Chinese Association of Automation, eds. Proceedings of the China Automation Association Conference, 2009 Nov 1-3, Hangzhou, Zhejiang University, 2009. 88-95

32 Wang Y C, Liu J G, Li B. A-B autonomy of a shape-shifting robot 'AMOEBA-I' for USAR. In: Behnam M, ed. Climbing and Walking Robots. Croatia: InTech, 2010. 425-444

33 Zhu H Y, Niu T F, Shen L C, et al. State of the art and trends of autonomous control of UAV systems (in Chinese). J Nation U Defense Tech, 2010, 32: 115-120

34 Ren M, Huo X H. Asynchronous double-precision windows based unmanned aerial vehicle real-time path planning. Sci China Inf Sci, 2010, 53: 215-222

35 Zhang X Y, Duan H B, Yu Y X. Receding horizon control for multi-UAVs close formation control based on differential evolution. Sci China Inf Sci, 2010, 53: 223-235

36 Duan H B, Liu S Q. Unmanned air/ground vehicles heterogeneous cooperative techniques: Current status and prospects. Sci China Tech Sci, 2010, 53: 1349-1355

37 Zou Q J, Zhang R B. Research on adjustable autonomy of unmanned system (in Chinese). Mini-micro Syst, 2009, 30: 2254-2258

38 Wu L Z, Niu Y F, Zhu H Y, et al. Modeling and characterizing of unmanned aerial vehicles autonomy (in Chinese). In: Meng Q H, Max H K, Jia L, et al., eds. Proceedings of the 8th World Congress on Intelligent Control and Automation, 2010 Jul 7-9, Jinan, China. IEEEXplore Press, 2010. 2284-2288

39 Wang Y C, Liu J G. Bio-inspired energy autonomy for robot (in Chinese). In: Information science editorial board of the 10000 selected problems, eds. 10000 Selected Problems in Sciences (Information Science). Beijing: Science Press, 2011. 692-693

40 Huntsberger T, Aghazarian H, Castano A, et al. Intelligent autonomy for unmanned sea surface and underwater vehicles. In: Association for Unmanned Vehicle Systems International (AUVSI), eds. Proceedings of the AUVSI Unmanned Systems, 2008 Jun 10-12, San Diego, California. Curran Associates, Inc Press. 2009, 111-123

41 Nickles M, Rovatsos M, Weiss G, eds. Autonomy 2003. Heidelberg: Springer, 2004. 17-19

42 Reed N E. A user controlled approach to adjustable autonomy. In: Proceedings of the 38th International Conference on System Sciences, 2005 Jan 3-6, Hawaii, IEEE Computer Society Press, 2005, 295-304

43 Committee on Autonomous Vehicles in Support of Naval Operations, National Research Council. Autonomous Vehicles in Support of Naval Operations. Washington DC: the National Academy of Sciences Press, 2005

44 Sheridan T B. Automation, authority and angst - revisited. In: Human Factors Society, eds. Proceedings of the Human Factors Society 35th Annual Meeting, 1991 Sep 2-6, San Francisco, California. Human Factors \& Ergonomics Society Press. 1991, 18-26

45 Parasoraman R, Sheridan T B, Wickens C D.A model for types and levels of human interaction with automation. IEEE T Syst Man Cy A, 2000, 30: 286-297

46 Larry A Y, Jeffrey A Y, Mark D G. System analysis applied to autonomy: Application to high-altitude long-endurance remotely operated aircraft. In: Proceedings of the AIAA Infotech Aerospace Conference, 2005 Sep 1-3, Seattle, 22-31

47 Cleary M, Abramson M, Adams M B, et al. Metrics for embedded collaborative intelligent systems. Technical report, Charles Stark Draper Laboratory, 2000

48 Curtin T, Crimmins D, Curcio J, et al. Autonomous underwater vehicles: Trends and transformations. Mar Technol Soc J, 2005, 39: 65-75 
49 Fleming M, Cohen R. A utility-based theory of initiative in mixed-initiative systems. In: Proceedings of The IJCAI-01 Workshop on Autonomy, Delegation, and Control: Interacting with Autonomous Agents, 2001 Aug 6-8, Seattle, 58-65

50 Brainov S, Hexmoor H. Quantifying relative autonomy in multiagent interaction. In: Multiagent Systems, Artificial Societies, Simulated Organizations,eds. Proceedings of The IJCAI-01 Workshop on Autonomy, Delegation, and Control: Interacting with Autonomous Agents, 2001 Aug 6-8, Seattle, 2003, 7: 55-73

51 Barber K, Martin C, Reed N, et al. Dimensions of adjustable autonomy. In: Kowalczyk R, Loke S W, Reed N, et al, eds. Advances in Artificial Intelligence: PRICAI 2000 Workshop Reader. Berlin: Springer, 2011. 353-361

52 Goodrich M A, Crandall J W, Stimpson J R. Neglect tolerant teaming: Issues and dilemmas. In: Alto P, ed. Proceedings of the 2003 AAAI Spring Symposium on Human Interaction with Autonomous Systems in Complex Environments, 2003 Mar 24-26, Stanford, CA, AAAI Press. 2003, 111-119

53 Clough B T. Metrics, schmetrics! How the heck do you determine a UAV's autonomy anyway? In: Proceedings of the Performance Metrics for Intelligent Systems Workshop, 2002 Aug 13-15, Gaithersburg, 12-20

54 Jochem T M, Pomerleau D A, Thorpe C E. Vision-based neural network road and intersection detection and traversal. In: Proceedings of the IEEE Conference on Intelligent Robots and Systems, 1995 Aug 5-9, Pittsburgh, 344-349

55 Doboli A, Curiac D, Pescaru D, et al. Cities of the future: Employing wireless sensor networks for efficient decision making in complex environments. Technical Report, State University of New York at Stony Brook, 2008

56 Zhuang Y, Chen D, Wang W, et al. Status and development of natural scene understanding for vision-based outdoor moblie robot (in Chinese). Acta Automat Sin, 2010, 36: 1-11

57 Jing X J,Wang Y C, Tan D L. Artificial coordinating field and its application to motion planning of robots in uncertain dynamic environments. Sci China Tech Sci, 2004, 47: 577-594

58 Liu J G, Ma S G, Wang Y C, et al. Network-based reconfiguration routes for a self-reconfigurable robot. Sci China Inf Sci, 2008, 51: $1532-1546$

59 Zhuang Y, Gu M W, Wang W, et al. Multi-robot cooperative localization based on autonomous motion state estimation and laser data interaction. Sci China Inf Sci, 2010, 53: 2240-2250

$60 \mathrm{Gu} F$, He Y Q, Han J D, et al. An active cooperative observation method for multi-robots in three dimensional environments (in Chinese). Acta Automat Sin, 2010, 36: 1443-1453

$61 \mathrm{Li} \mathrm{Z} \mathrm{Q,} \mathrm{Ma} \mathrm{S} \mathrm{G,} \mathrm{Li} \mathrm{B,} \mathrm{et} \mathrm{al.} \mathrm{Analysis} \mathrm{of} \mathrm{the} \mathrm{constraint} \mathrm{relation} \mathrm{be-}$ tween ground and selfadaptive mobile mechanism of a transformable wheel-track robot. Sci China Tech Sci, 2011, 54: 610-624

$62 \mathrm{Lu} \mathrm{Z} \mathrm{L,} \mathrm{Ma} \mathrm{S} \mathrm{G,} \mathrm{Li} \mathrm{B,} \mathrm{et} \mathrm{al.} \mathrm{Gaits-transferable} \mathrm{CPG} \mathrm{controller} \mathrm{for} \mathrm{a}$ snake-like robot. Sci China Inf Sci, 2008, 51: 293-305

63 Liu J G, Wang Y C, Li B, et al. Center-configuration selection technique for the reconfigurable modular robot. Sci China Inf Sci, 2007, 50: 697-910

64 Duan H B, Shao S, Su B W, et al. New development thoughts on the bio-inspired intelligence based control for unmanned combat aerial vehicle. Sci China Tech Sci, 2010, 53: 2025-2031

65 Song D L, Qi J T, Han J D, et al. Model identification and active modeling control for rotor fly-robot: Theory and experiment (in Chinese). Acta Automat Sin, 2011, 37: 480-495

66 Bowen D G, MacKenzie S C. Autonomous collaborative unmanned vehicles: Technological drivers and constraints. Technical Report, Defence R\&D Canada, 2003

$67 \mathrm{Xu} \mathrm{Y} \mathrm{R,} \mathrm{Su} \mathrm{Y} \mathrm{M,} \mathrm{Pang} \mathrm{Y} \mathrm{J.} \mathrm{Expectation} \mathrm{of} \mathrm{the} \mathrm{development} \mathrm{in} \mathrm{the}$ technology on ocean space intelligent unmanned vehicles (in Chinese). Chin J Ship Res, 2006, 1: 1-4

68 John C M. Technology readiness levels: A white paper. Technical Report, Advanced Concepts Office, Office of Space Access and Technology, NASA, 1995

69 Hobson B. A technology maturity measurement system for the department of national defence: The TML System. Technical Report, Defence R\&D Canada-Atlantic, 2006

70 Guo D Q. TRL Based Technology Maturity Model and Assessment Research (in Chinese). Master Dissertation. Changsha: National University of Defense Technology, 2010

71 James W B. A suite of tools for technology assessment. Proceedings of the AFRL Technology Maturity Conference, 2007 Sept 11-13, Virginia Beach, VA, 1123-1129

72 Qian D, Cui L, Xiao C M. Technology readiness level evaluation method and application to underwater weapon (in Chinese). Torpedo Tech, 2006, 14: 1-7

73 Chen Y B, Huang Y H. Missile technology maturity assessment using TRL method (in Chinese). Sci Tech Res, 2007, 8: 3-4

74 Zhu Y L. Develop aerospace technology maturity research (in Chinese). Aerosp Ind Manag, 2008, 5: 10-13

75 Caroline P G, Suzanne G, Jeannine S, et al. Using the technology readiness levels scale to support technology management in the DoD's ATD/STO Environments. Technical Report, Carnegie Mellon University, 2002

76 Nolte W L, Kennedy B C, Dziegiel R J J. Technology readiness level calculator. In: NDIA Systems Engineering Conference, 2003 Oct 20-22. Presentation available from http://lincoln.gsfc.nasa.gov/trl/ Nolte2003.pdf

Open Access This article is distributed under the terms of the Creative Commons Attribution License which permits any use, distribution, and reproduction in any medium, provided the original author(s) and source are credited. 